\title{
Conformal profiles in the Hilhorst-van Leeuwen model
}

\author{
D Karevski†, L Turban† and F Iglóił §§ \\ $\nmid$ Laboratoire de Physique des Matériaux, Université Henri Poincaré (Nancy I), \\ BP 239, F-54506 Vandœuvre lès Nancy Cedex, France \\ $\ddagger$ Research Institute for Solid State Physics and Optics, PO Box 49, H-1525 \\ Budapest, Hungary \\ $\S$ Institute of Theoretical Physics, Szeged University, H-6720 Szeged, Hungary
}

Received 22 December 1999

\begin{abstract}
We study the critical energy and magnetization profiles for the Ising quantum chain with a marginal extended surface perturbation of the form $A / y$, $y$ being the distance from the surface (Hilhorst-van Leeuwen model). For weak local couplings, $A<A_{\mathrm{c}}$, the model displays a continuous surface transition with $A$ dependent exponents, whereas, for $A>A_{\mathrm{c}}$, there is surface order at the bulk critical point. If conformal invariance is assumed to hold with such marginal perturbations, it predicts conformal profiles with the same scaling form as for the unperturbed quantum chain, with marginal surface exponents replacing the unperturbed ones. The results of direct analytical and numerical calculations of the profiles confirm the validity of the conformal expressions in the regimes of second- and first-order surface transitions.
\end{abstract}

\section{Introduction}

The methods of conformal invariance have been extensively used in twodimensional (2D) critical systems, first to study the bulk critical properties [1] but also, very soon after, to explore the local critical behaviour at surfaces [2] or near line defects [3]. Although translation invariance is broken in the transverse direction for such defective systems, conformal invariance is preserved. In the case of the line defect, the spectrum-generating algebra was identified as the $U(1)$ Kac-Moody algebra [4]. More surprisingly, some aspects of conformal invariance, such as gapexponent relations, seem to hold for marginal extended perturbations, provided the shape of the perturbation is properly transformed [5-10].

Extended perturbations were first considered at the surface of the semi-infinite 2D Ising model by Hilhorst and van Leeuwen (HvL) [11]. This problem, as well as its extensions to linear and radial defects in the bulk, became an active field of research during the years that followed [12-20]. Different approaches, either numerical or analytical, have been used to elucidate the influence of such perturbations on the local critical behaviour (see [21] for a review). 
Surface extended perturbations correspond to smoothly varying inhomogeneties for which the nearest-neighbour coupling, at a distance $y$ from the surface, deviates from its bulk value by a quantity $A y^{-\kappa}$. When the decay exponent $\kappa$ is equal to the scaling dimension of the couplings, the perturbation is marginal and the local critical exponents vary continuously with the perturbation amplitude $A$ [22-24]. For a sufficiently strong enhancement of the perturbation amplitude $A$, the surface remains ordered at the bulk critical point. When $\kappa$ is larger than the marginal value, the perturbation decreases sufficiently fast and the critical properties remain unchanged. On the other hand, for a value of $\kappa$ smaller than the critical one, the system is driven to a new surface fixed point, at which power laws are replaced by stretched exponentials for $A<0$ whereas the surface remains ordered at criticality when $A>0$.

In the present paper, we consider the off-diagonal profiles [25] of the energy and magnetization densities for the 2D Ising model with a marginal HvL surface extended defect. We work in a strip geometry resulting from a logarithmic conformal mapping of the half-space. We treat the problem in the extreme anisotropic limit [26], where the 2D classical Ising model corresponds to a one-dimensional (1D) Ising quantum chain in a transverse field. The energy density profiles are obtained analytically, working in the continuum limit, whereas the magnetization profiles are studied numerically. The results are compared with the conformal predictions in the different regimes.

The paper is organised as follows. In section 2, we review briefly the conformal results for the surface correlation functions in the presence of a marginal $\mathrm{HvL}$ perturbation and the resulting off-diagonal profiles in the strip geometry. In section 3, we present the HvL model in the extreme anisotropic limit and the diagonalization of the associated Hamiltonian in the continuum limit. The off-diagonal profiles are calculated in section 4 . The results are discussed in section 5 .

\section{Marginal extended perturbations and conformal invariance}

Following [10] let us first consider the behaviour, under the infinitesimal special conformal transformation $z^{\prime}=z+\epsilon z^{2}$, of a marginal inhomogeneous coupling of the form $\Delta(\rho, \theta)=A \rho^{-y_{\chi}} f(\theta)$, conjugate to the field $\chi$. We use polar coordinates, $z=\rho \exp (\mathrm{i} \theta)$, and $y_{\chi}$ is the scaling dimension of $\Delta$. Up to $\mathrm{O}(\epsilon)$ we have

$$
\rho^{\prime}=\rho+\epsilon \rho^{2} \cos \theta \quad \theta^{\prime}=\theta+\epsilon \rho \sin \theta
$$

so that the half-plane, with $0 \leq \theta \leq \pi$, is mapped onto itself. The local dilatation factor is $b(\rho, \theta)=\left|\mathrm{d} z^{\prime} / \mathrm{d} z\right|^{-1}=1-2 \epsilon \rho \cos \theta$ and leads to the following transformed perturbation:

$\Delta\left(\rho^{\prime}, \theta^{\prime}\right)=(1-2 \epsilon \rho \cos \theta)^{y_{\chi}} A \frac{f(\theta)}{\rho^{y_{\chi}}}=\left[1-\epsilon \rho \sin \theta\left(y_{\chi} \cot \theta+\frac{\mathrm{d} \ln f}{\mathrm{~d} \theta}\right)\right] A \frac{f\left(\theta^{\prime}\right)}{\rho^{\prime y_{\chi}}}$.

The perturbation is invariant when the coefficient of $\epsilon$ vanishes, i.e., when $\Delta(\rho, \theta)=$ $A|\rho \sin \theta|^{-y_{\chi}}$, which is just the form of the marginal HvL perturbation.

If conformal invariance holds with such a marginal perturbation, the two-point correlation functions satisfy the same set of partial differential equations as the unperturbed ones [2]. 
The transformation properties of the two-point functions under the infinitesimal special conformal transformation imply the following scaling behaviour at criticality [2]:

$$
G_{\phi \phi}\left(x_{1}-x_{2}, y_{1}, y_{2}\right)=\left(y_{1} y_{2}\right)^{-x_{\phi}} g_{\phi}(\omega) \quad \omega=\frac{\left(x_{1}-x_{2}\right)^{2}+\left(y_{1}-y_{2}\right)^{2}}{y_{1} y_{2}}
$$

where $x(y)$ is the direction parallel (perpendicular) to the surface and $x_{\phi}$ the bulk scaling dimension of the field $\phi$. Using polar coordinates and taking the limit $\rho_{1} \gg \rho_{2}$, simple scaling considerations lead to

$$
G_{\phi \phi}\left(\rho_{1}, \rho_{2}, \theta_{1}, \theta_{2}\right) \sim\left(\rho_{1} \rho_{2}\right)^{-x_{\phi}}\left(\frac{\rho_{1}}{\rho_{2}}\right)^{-x_{\phi}^{\mathrm{s}}}\left(\sin \theta_{1} \sin \theta_{2}\right)^{x_{\phi}^{\mathrm{s}}-x_{\phi}}
$$

where $x_{\phi}^{\mathrm{s}}$ is the surface scaling dimension of $\phi$. Using the logarithmic conformal transformation, $w=(L / \pi) \ln z$, with $z=x+\mathrm{i} y=\rho \exp (\mathrm{i} \theta)$ and $w=u+\mathrm{i} v$, the half-plane, $y>0$, with uniform boundary conditions is mapped onto a strip $(-\infty<u<+\infty, 0<v<L)$ with the same boundary conditions on both edges. The correlation functions in the two geometries are related under the conformal mapping so that

$$
G_{\phi \phi}\left(w_{1}, w_{2}\right)=\left[b\left(z_{1}\right) b\left(z_{2}\right)\right]^{x_{\phi}} G_{\phi \phi}\left(z_{1}, z_{2}\right)
$$

where $b(z)=|\mathrm{d} w / \mathrm{d} z|^{-1}$ is the local dilatation factor. Thus one obtains the well known result for the two-point function in the strip geometry [27]

$G_{\phi \phi}\left(u_{1}-u_{2}, v_{1}, v_{2}\right) \sim\left(\frac{\pi}{L}\right)^{2 x_{\phi}}\left[\sin \left(\frac{\pi v_{1}}{L}\right) \sin \left(\frac{\pi v_{2}}{L}\right)\right]^{x_{\phi}^{\mathrm{s}}-x_{\phi}} \exp \left[-\frac{\pi}{L} x_{\phi}^{\mathrm{s}}\left(u_{1}-u_{2}\right)\right]$

when $u_{1} \gg u_{2}$.

This result is expected to apply with a marginal extended perturbation, provided the shape of the perturbation is also transformed according to the logarithmic mapping. Details will be given in the next section. Then $x_{\phi}^{\mathrm{s}}$ is the continuously varying surface scaling dimension of $\phi$ associated with the marginal defect.

In the strip geometry, the row-to-row transfer operator $\mathcal{T}$ of the isotropic classical system can be used to expand the correlation function $G_{\phi \phi}$ over its eigenstates $|n\rangle$ such that $\mathcal{T}|n\rangle=\exp \left(-E_{n}\right)|n\rangle$. This leads to

$G_{\phi \phi}\left(u_{1}-u_{2}, v_{1}, v_{2}\right)=\sum_{n>0}\left\langle 0\left|\hat{\phi}\left(v_{1}\right)\right| n\right\rangle\left\langle n\left|\hat{\phi}\left(v_{2}\right)\right| 0\right\rangle \exp \left[-\left(E_{n}-E_{0}\right)\left(u_{1}-u_{2}\right)\right]$

where $|0\rangle$ is the eigenstate corresponding to the largest eigenvalue of the transfer operator and $\hat{\phi}$ the operator associated with the classical field $\phi$. In the limit $u_{1} \gg u_{2}$, the sum is dominated by the eigenstate $|\phi\rangle$ with the largest eigenvalue $\exp \left(-E_{\phi}\right)$ and a non-vanishing matrix element $\langle 0|\hat{\phi}| \phi\rangle$ so that finally one obtains:

$G_{\phi \phi}\left(u_{1}-u_{2}, v_{1}, v_{2}\right) \simeq\left\langle 0\left|\hat{\phi}\left(v_{1}\right)\right| \phi\right\rangle\left\langle\phi\left|\hat{\phi}\left(v_{2}\right)\right| 0\right\rangle \exp \left[-\left(E_{\phi}-E_{0}\right)\left(u_{1}-u_{2}\right)\right]$.

A comparison to equation (2.6) allows us to deduce the off-diagonal profile for $\phi$ in the strip geometry [25]:

$$
\phi_{o d}(v)=\langle 0|\hat{\phi}(v)| \phi\rangle \sim\left(\frac{\pi}{L}\right)^{x_{\phi}}\left[\sin \left(\frac{\pi v}{L}\right)\right]^{x_{\phi}^{\mathrm{s}}-x_{\phi}} .
$$


One also recovers the gap-exponent relation [27]

$$
E_{\phi}-E_{0}=\frac{\pi}{L} x_{\phi}^{\mathrm{s}}
$$

which is valid under this form for the isotropic strip.

Off-diagonal profiles contain singular contributions only, which facilitates the scaling analysis in numerical calculations. Furthermore, an external symmetrybreaking field is not needed to obtain a non-vanishing order parameter density. These profiles also give an information about the surface critical behaviour since equation (2.9) with $v \ll L$ leads to $\phi_{\text {od }}(v) \sim L^{-x_{\phi}^{s}}$.

One may note that the diagonal profile

$$
\phi(v) \sim\left[\sin \left(\frac{\pi v}{L}\right)\right]^{-x_{\phi}}
$$

obtained in [28] for the order parameter, with symmetric fixed boundary conditions, is formally recovered by setting $x_{\phi}^{\mathrm{s}}=0$ in equation (2.9), i.e., the scaling dimension of the order parameter at the extraordinary surface transition.

\section{Diagonalization of the HvL Ising quantum chain in the continuum limit}

Let us start with the classical HvL Ising model on a semi-infinite square lattice with nearest-neighbour couplings

$$
\Delta K_{2}(y)=K_{2}(y)-K_{2}(\infty)=A y^{-\kappa} \quad K_{2}(\infty)=K_{2}
$$

in the direction perpendicular to the surface and constant couplings, $K_{1}$, in the direction parallel to the surface. Hereafter we consider the marginal situation where $\kappa=1 / \nu=1$ for the $2 \mathrm{D}$ Ising model. Under the logarithmic conformal mapping, the marginal perturbation in the half-plane transforms into

$$
\Delta K_{2}(v)=A\left[\frac{L}{\pi} \sin \left(\frac{\pi v}{L}\right)\right]^{-1}
$$

in the strip geometry where $0<v<L$.

The spectrum of the row-to-row transfer matrix has been obtained analytically [5] in the extreme anisotropic limit where $K_{2} \rightarrow 0, K_{1} \rightarrow \infty$ while $K_{2} / K_{1}^{*}=\lambda$ remains constant [26]. Here $K_{1}^{*}$ is the dual coupling such that $\tanh K_{1}^{*}=\exp \left(-2 K_{1}\right)$ and self-duality allows to locate the critical point at $\lambda_{\mathrm{c}}=1$. The amplitude $A \rightarrow 0$, too, and thus can be parametrized as $A=\alpha K_{1}^{*}$.

In the extreme anisotropic limit, the fluctuations are anisotropic with a correlation length ratio given by [29]

$$
\frac{\xi_{2}}{\xi_{1}}=\frac{\cosh \left(2 K_{2}\right)}{\cosh \left(2 K_{1}\right)} \simeq 2 K_{1}^{*}
$$

Isotropy is restored by rescaling the lattice parameter $a_{1}$ in the time direction to $a_{1}=2 K_{1}^{*}$ measured in units of $a_{2}$ in the transverse direction. The row-to-row transfer operator, which can be written as $\mathcal{T}_{a_{1}}=\exp \left(-2 K_{1}^{*} \mathcal{H}\right)$, has to be applied 
$1 / a_{1}$ times in order to ensure a transfer by one unit of $a_{2}$ on the rescaled isotropic system. Thus one may identitfy the transfer operator $\mathcal{T}$ of the isotropic system to $\exp (-\mathcal{H})$ where $\mathcal{H}$ is the Hamiltonian of the Ising quantum chain in a transverse field such that $\mathcal{H}|n\rangle=E_{n}|n\rangle$.

The critical Hamiltonian reads

$$
\mathcal{H}=-\frac{1}{2}\left[\sum_{l=1}^{L-1} \lambda_{l} \sigma_{l}^{x} \sigma_{l+1}^{x}+\sum_{l=1}^{L} \sigma_{l}^{z}\right]
$$

where the $\sigma$ s are the Pauli spin operators, and the couplings $\lambda_{l}$ are given by

$$
\lambda_{l}=1+\alpha\left[\frac{L}{\pi} \sin \left(\frac{\pi l}{L}\right)\right]^{-1} .
$$

After a Jordan-Wigner transformation [30], the Hamiltonian (3.4) becomes a quadratic form in fermion creation and annihilation operators. It is diagonalized through a canonical transformation $[31,32]$ leading to

$$
\mathcal{H}=\sum_{k} \Lambda_{k}\left(\eta_{k}^{\dagger} \eta_{k}-\frac{1}{2}\right)
$$

The $\eta_{k}^{\dagger} \mathrm{s}\left(\eta_{k} \mathrm{~s}\right)$ are diagonal fermion creation (anihilation) operators and the $\Lambda_{k} \mathrm{~s}$ are the energies of the fermionic excitations. They satisfy the following set of equations:

$$
(\mathbf{A}+\mathbf{B}) \phi_{k}=\Lambda_{k} \boldsymbol{\psi}_{k} \quad(\mathbf{A}-\mathbf{B}) \boldsymbol{\psi}_{k}=\Lambda_{k} \phi_{k}
$$

where $\phi_{k}$ and $\boldsymbol{\psi}_{k}$ are normalized eigenvectors. Working with the $\boldsymbol{\psi}_{k}$ s such that $(\mathbf{A}+\mathbf{B})(\mathbf{A}-\mathbf{B}) \boldsymbol{\psi}_{k}=\Lambda_{k}^{2} \boldsymbol{\psi}_{k}$ and changing $\psi_{k}(l)$ into $(-1)^{l} \psi_{k}(l)$, one is led to the eigenvalue problem,

$$
-\lambda_{l-1} \psi_{k}(l-1)+\left[\lambda_{l}^{2}+1\right] \psi_{k}(l)-\lambda_{l} \psi_{k}(l+1)=\Lambda_{k}^{2} \psi_{k}(l) \quad l=1, L
$$

with the boundary conditions

$$
\psi_{k}(0)=0 \quad \lambda_{l} \psi_{k}(L)-\psi_{k}(L+1)=0 .
$$

To study the low-energy spectrum, one may solve the finite-difference equation (3.8), with $\lambda_{l}$ given by (3.5), by working in the continuum limit [5]. To second order in $1 / L$, using the continuum variable $z=\pi l / L$, the difference equation is replaced by the differential equation

$$
\psi_{k}^{\prime \prime}(z)+\left[\left(\frac{L \Lambda_{k}}{\pi}\right)^{2}-\frac{\alpha^{2}-\alpha \cos z}{\sin ^{2} z}\right] \psi_{k}(z)=0
$$

with the boundary conditions

$$
\left.\psi_{k}(z)\right|_{z=0}=0 \quad \psi_{k}^{\prime}(z)-\left.\frac{\alpha}{\sin z} \psi_{k}(z)\right|_{z=\pi}=0 .
$$


Using the change of function $\psi_{k}(z)=\cos ^{-\alpha}(z / 2) \sin ^{1-\alpha}(z / 2) f_{k}(t)$ where $t=$ $\sin ^{2}(z / 2),(3.11)$ becomes a hypergeometric differential equation. Its general solution is given by [5]

$$
f_{k}(t)=C_{1} F(a, b ; c ; t)+C_{2} t^{1-c} F(a-c+1, b-c+1 ; 2-c ; t)
$$

where

$$
a=-\alpha+\frac{1}{2}+\frac{\Lambda_{k} L}{\pi} \quad b=-\alpha+\frac{1}{2}-\frac{\Lambda_{k} L}{\pi} \quad c=\frac{3}{2}-\alpha .
$$

Due to the boundary conditions in equation (3.11), one has to separately consider two different regimes:

(i) When $\alpha<\frac{1}{2}$, the condition at $z=0$ imposes $C_{2}=0$ while the quantization of the spectrum,

$$
\Lambda_{k}=\frac{\pi}{L}\left(\frac{1}{2}-\alpha+k\right) \quad k=0,1,2, \ldots
$$

follows from the condition at $z=\pi$. The spectrum exhibits a conformal towerlike structure but, to our knowledge, until now no underlying algebra has been identified. Using the gap-exponent relation in equation (2.10), one deduces the critical dimension of the magnetization from $E_{\sigma}-E_{0}=\Lambda_{0}$ and the critical dimension of the energy density from $E_{\epsilon}-E_{0}=\Lambda_{0}+\Lambda_{1}$. Thus we have

$$
x_{\mathrm{m}}^{\mathrm{s}}=\frac{1}{2}-\alpha \quad x_{\mathrm{e}}^{\mathrm{s}}=2-2 \alpha .
$$

These values are in agreement with the result obtained on a semi-infinite system for the magnetic exponent [11] and through finite-size scaling on a strip for both exponents [19], in the extreme anisotropic limit.

Using equations (3.12)-(3.14), one obtains the eigenfunctions

$$
\psi_{k}(z) \sim \frac{1}{L^{1 / 2}} \frac{\sin ^{1-\alpha}(z / 2)}{\cos ^{\alpha}(z / 2)} \frac{k !}{(3 / 2-\alpha)_{k}} P_{k}^{\left(\frac{1}{2}-\alpha,-\frac{1}{2}-\alpha\right)}[\cos z]
$$

where $P_{k}^{(\beta, \gamma)}[z]$ is the Jacobi polynomial of degree $k$ [33]. The prefactor, $1 / L^{1 / 2}$, follows from the normalization.

(ii) When $\alpha>\frac{1}{2}$, the boundary conditions lead to $C_{1}=0$ and

$$
\Lambda_{0}=\mathrm{O}\left(L^{-2 \alpha}\right) \quad \Lambda_{k}=\frac{\pi}{L}\left(\frac{1}{2}+\alpha+k-1\right) \quad k=1,2,3, \ldots
$$

Again a tower-like structure appears. One has to notice that the differential equation (3.10) together with (3.11) gives $\Lambda_{0}=0$ since, in the continuum limit, the excitation spectrum is calculated to the first order in $1 / L$. But, as shown in [5], the correct $\mathrm{O}\left(L^{-2 \alpha}\right)$ behaviour can be extracted from the difference equation (3.8) using the trial eigenvector $\psi_{0}(l) \sim \tan ^{\alpha}(\pi l / 2 L)$ given by (3.10) with $\Lambda_{0}=0$.

The anomalous behaviour of the first excitation is linked to the persistence of a spontaneous surface magnetization at the critical point for $\alpha>\frac{1}{2}[12,15]$. The surface order is due to the enhancement of the couplings near the surface which 
overcompensates the missing bond. The surface displays a first-order transition at the bulk critical point with a jump of the surface magnetization to zero when $\lambda<1$ since, considering the classical picture, the 1D surface cannot remain ordered when the bulk is disordered.

From the gap-exponent relation (2.10), the following critical indices are deduced:

$$
x_{\mathrm{m}}^{\mathrm{s}}=0 \quad x_{\mathrm{e}}^{\mathrm{s}}=\frac{1}{2}+\alpha .
$$

The eigenfunctions read

$$
\begin{aligned}
& \psi_{0}(z) \sim \frac{1}{L^{\alpha}} \tan ^{\alpha}\left(\frac{z}{2}\right) \\
& \psi_{k \geq 1}(z) \sim \frac{1}{L^{1 / 2}} \tan ^{\alpha}\left(\frac{z}{2}\right) \cos ^{1+2 \alpha}\left(\frac{z}{2}\right) F\left[-k+1, k+2 \alpha ; \frac{1}{2}+\alpha ; \sin ^{2}\left(\frac{z}{2}\right)\right]
\end{aligned}
$$

where the hypergeometric function, $F$, reduces to a polynomial of order $k-1$. The anomalous normalization of $\psi_{0}(z)$ is a consequence of the spontaneous surface order.

In analogy with surface transitions in homogeneous systems, (i) is a regime of ordinary surface transition. For (ii) we use the name of first-order surface transition.

\section{Off-diagonal conformal profiles}

\subsection{Energy-density profiles}

First we consider the energy-density profile for which we derive exact expressions. The off-diagonal profile is given by the matrix element $e(l)=\left\langle 0\left|\sigma_{l}^{z}\right| \epsilon\right\rangle$ where $|\epsilon\rangle=\eta_{1}^{\dagger} \eta_{0}^{\dagger}|0\rangle$ is the lowest excited state with an even number of excitations. Writing $\sigma^{z}$ in terms of diagonal fermions, in the continuum limit, one obtains [19]

$$
e(z)=\psi_{0}(z) \psi_{1}(\pi-z)+\psi_{0}(\pi-z) \psi_{1}(z) .
$$

(i) At the ordinary surface transition, $\alpha<\frac{1}{2}$, using equation (3.16) with $k=0$ and $k=1$, a straightforward calculation leads to the following expression:

$$
e(z)=\frac{A}{L}(\sin z)^{1-2 \alpha}
$$

where $A$ is a constant amplitude. This expression agrees with the conformal result in equation (2.9) since $z=\pi l / L$, the bulk scaling dimension of the energy is $x_{\mathrm{e}}=1$ for the Ising model and the surface one is $x_{\mathrm{e}}^{\mathrm{s}}=2-2 \alpha$ according to (3.15).

(ii) At the border line, $\alpha=\frac{1}{2}$, the surface magnetization vanishes logarithmically [12] and the first excitation scales normally as $1 / L$. One may use equation (4.1) to calculate the energy profile. In this case both (3.16) and (3.19) give the same solution. In particular $\psi_{0}(z) \sim L^{-1 / 2} \tan ^{1 / 2}(z / 2)$ and $\psi_{1}(z) \sim$ 


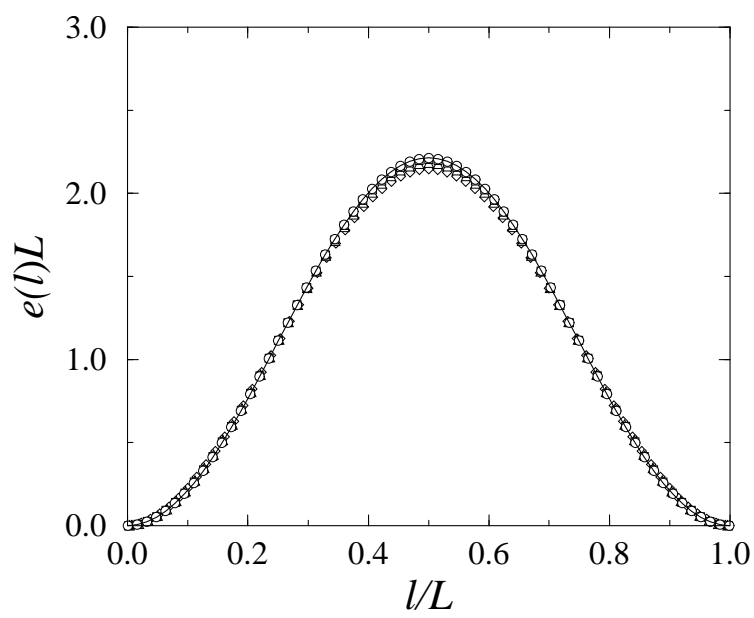

Figure 1. Rescaled energy-density profile $e(z)$ in the regime of ordinary surface transition with $\alpha=-\frac{1}{2}$ for chain sizes $L=2^{6}$ (diamond), $2^{7}$ (triangle), $2^{8}$ (square) and $2^{9}$ (circle). The conformal profile (full curve) is shown for comparison.

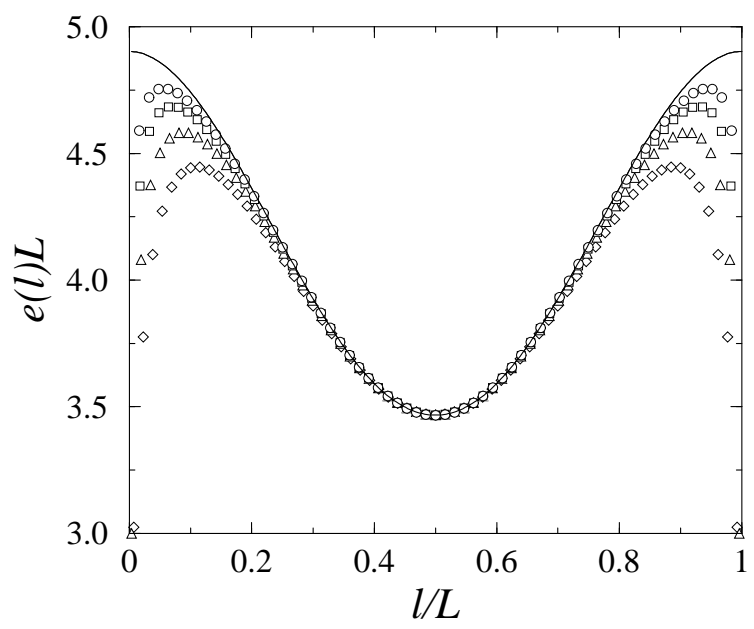

Figure 2. Rescaled energy-density profile $e(z)$ in the regime of first-order surface transition with $\alpha=1$ for chain sizes $L=2^{6}$ (diamond), $L=2^{7}$ (triangle), $2^{8}$ (square) and $2^{9}$ (circle). The result obtained in the continuum approximation (full curve) is shown for comparison.

$L^{-1 / 2} \tan ^{1 / 2}(z / 2) \cos ^{2}(z / 2)$. Inserting these eigenvectors into (4.1) one obtains a constant profile,

$$
e(z)=\frac{D}{L}
$$




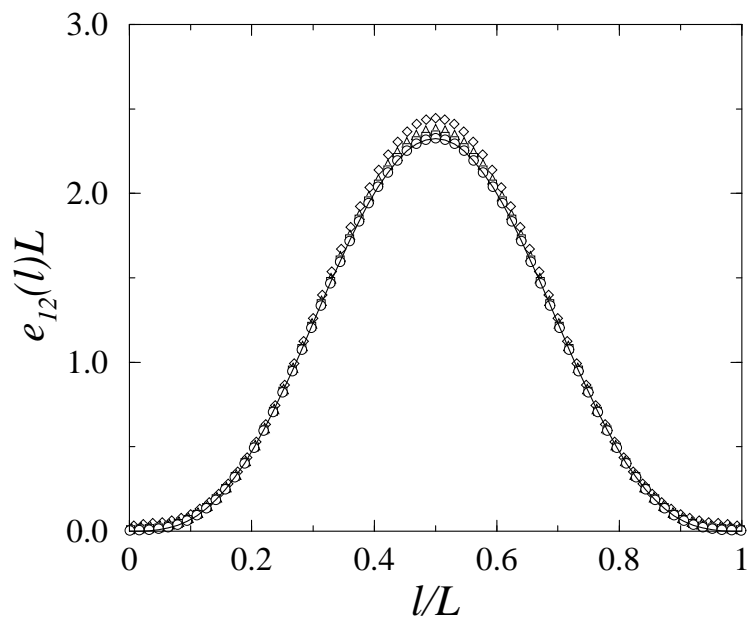

Figure 3. Rescaled energy-density profile $e_{12}(z)$ in the regime of first-order surface transition with $\alpha=1$ for chain sizes $L=2^{6}$ (diamond), $L=2^{7}$ (triangle), $2^{8}$ (square) and $2^{9}$ (circle). The conformal profile (full curve) is shown for comparison.

in the continuum limit. This may be traced to the fact that, at $\alpha=\frac{1}{2}$, the energy density has the same scaling dimension at the surface as in the bulk. In the energy sector the system does not feel anymore the surface and behaves asymptotically as a periodic one. The profile (4.3) is recovered using (2.9) with $x_{\mathrm{e}}^{\mathrm{s}}=x_{\mathrm{e}}=1$. Actually, the numerical data display logarithmic corrections. Good fits of the finite-size results were obtained with the functional form $e(z)=A_{0}+A_{1} \ln (\sin z)$ where $A_{0} \gg A_{1}$.

(iii) For $\alpha>\frac{1}{2}$, i.e., in the first-order regime, equation (4.1) leads to the following expression for the energy density:

$$
e(z)=\frac{B}{L^{1 / 2+\alpha}}\left[\cos ^{2 \alpha+1}\left(\frac{z}{2}\right)+\sin ^{2 \alpha+1}\left(\frac{z}{2}\right)\right] .
$$

Near the surface $e(z)$ scales as $L^{-(1 / 2+\alpha)}$, i.e., according to (3.18), the gapexponent relation is satisfied but the profile has a functional form which clearly disagrees with the conformal expression in (2.9). This is due to the localization near $z=\pi$ of the lowest eigenstate $\psi_{0}(z)$, with vanishing eigenvalue in the continuum limit. This anomalous behaviour is related to the persistence of surface order at criticality. The next two eigenstates are extended and have a normal scaling behaviour. Let us consider the corresponding off-diagonal energy profile,

$$
e_{12}(z)=\psi_{1}(z) \psi_{2}(\pi-z)+\psi_{1}(\pi-z) \psi_{2}(z)
$$

Inserting (3.19) with $k=1,2$ yields:

$$
e_{12}(z)=\frac{C}{L}(\sin z)^{2 \alpha+1}
$$


This is to be compared with (2.9) with $x_{\mathrm{e}}=1$ and the approriate surface scaling dimension, $\left(x_{\mathrm{e}}^{\mathrm{s}}\right)_{12}$, which is not given by (3.18) but can be deduced from the gapexponent relation (2.10) and reads:

$$
\left(x_{\mathrm{e}}^{\mathrm{s}}\right)_{12}=\frac{L}{\pi}\left(\Lambda_{1}+\Lambda_{2}\right)=2 \alpha+2 .
$$

Thus we find an agreement with the behaviour expected from conformal invariance, if we leave out the anomalous first excitation. This point is discussed further in the final section where a parallel is made with the analogous situation for the homogeneous system with fixed boundary conditions.

The energy density profiles obtained numerically in the two regimes using equation (4.1) for $\alpha<\frac{1}{2}$ and equations (4.1) and (4.5) for $\alpha>\frac{1}{2}$ are shown in figures $1-3$, respectively.

\subsection{Magnetization profiles}

The off-diagonal magnetization profile is given by the matrix element $m(l)=\left\langle 0\left|\sigma_{l}^{x}\right| \sigma\right\rangle$, where $|\sigma\rangle=\eta_{0}^{\dagger}|0\rangle$ is the lowest excited state in the odd sector of $\mathcal{H}$ with energy $E_{\sigma}$. Rewriting $\sigma_{l}^{x}$ in terms of diagonal fermions and using Wick's theorem, the local magnetization can be expressed as a determinant [34]

$$
m(l)=\left|\begin{array}{ccccc}
H_{1} & G_{11} & G_{12} & \ldots & G_{1 l-1} \\
H_{2} & G_{21} & G_{22} & \ldots & G_{2 l-1} \\
\vdots & \vdots & \vdots & & \vdots \\
H_{l} & G_{l 1} & G_{l 2} & \ldots & G_{l l-1}
\end{array}\right|
$$

with

$$
H_{j}=\phi_{0}(j) \quad G_{i j}=-\sum_{k} \phi_{k}(i) \psi_{k}(j)
$$

where the vectors $\phi_{k}$ and $\boldsymbol{\psi}_{k}$ are the eigenvectors defined in (3.7). Since $\sigma_{l}^{x}$ is nonlocal in terms of the diagonal fermion operators, $\eta_{k}^{\dagger}$ and $\eta_{k}$, the expression of $m(l)$ is much more complicated than the corresponding one (4.1) for the energy density and we had to study the behaviour of the magnetization profile numerically.

The rescaled profiles shown in figure 4, obtained for different values of $\alpha$, converge with increasing sizes towards the conformal expression which, according to (2.9) with $x_{\mathrm{m}}=\frac{1}{8}$ for the bulk magnetic exponent, takes the form

$$
m(z)=\frac{A}{L^{1 / 8}}(\sin z)^{x_{\mathrm{m}}^{\mathrm{s}}-1 / 8}
$$

where $z=\pi l / L$.

For $\alpha<\frac{1}{2}$, the critical exponent $x_{\mathrm{m}}^{\mathrm{s}}=\frac{1}{2}-\alpha$ has to be used. In particular, the value $\alpha=\frac{3}{8}$ corresponds to equal surface and bulk scaling dimensions, $x_{\mathrm{m}}^{\mathrm{s}}=x_{\mathrm{m}}=\frac{1}{8}$, and then (4.10) leads to a constant magnetization profile. The deviations near the surfaces in figure 4 , which are slowly decreasing with increasing sizes, are due to finite-size corrections.

In the first-order regime, $\alpha>\frac{1}{2}$, the critical profile has the same form as for the homogeneous system with fixed boundary conditions since $x_{\mathrm{m}}^{\mathrm{s}}=0$ in both cases. The rescaled profile is divergent at the surface in the continuum limit, the numerical data obtained on the discrete system display this tendency with increasing sizes. 


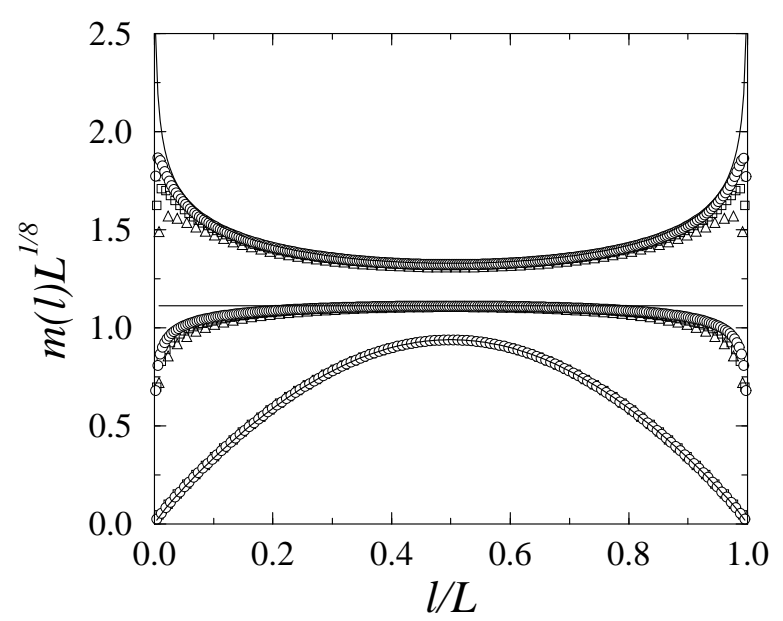

Figure 4. Rescaled magnetization profiles with $\alpha=\frac{3}{2}, \alpha=\frac{3}{8}$ and $\alpha=-\frac{1}{2}$ from top to bottom for chain sizes $2^{6}$ (triangle), $2^{7}$ (square) and $2^{8}$ (circle). The full curves give the corresponding conformal profiles.

\section{Discussion}

The anomalous behaviour of the energy-density profile in the first-order regime, $\alpha>\frac{1}{2}$, is linked to the localization, near $z=0$, of the state $\psi_{0}(z)$. The corresponding excitation, $\Lambda_{0}$, vanishes as $L^{-2 \alpha}$, i.e., faster than higher excitations which display the usual $L^{-1}$ scaling behaviour. The localization of the lowest eigenstate is itself related to the persistence of surface order at criticality. This suggests an analogy with the homogeneous system with symmetric fixed boundary conditions for which, similarly, the lowest excitation vanishes and corresponds to a mode which is completely localized at the surface. The energy spectrum is doubly degenerate and the first mode does not contribute to the conformal profile which is given by the matrix element $\left\langle 0\left|\sigma^{z}\right| \epsilon_{++}\right\rangle$, where $\left|\epsilon_{++}\right\rangle=\eta_{2}^{\dagger} \eta_{1}^{\dagger}|0\rangle$ is the first non-trivial even state [25]. The spectrum and eigenvectors are those of the dual chain with free boundary conditions. In the continuum limit, the energy-density profile is given by (2.9) with $x_{e}=1$ and $\left(x_{\mathrm{e}}^{\mathrm{s}}\right)_{++}=L / \pi\left(\Lambda_{1}+\Lambda_{2}\right)=2$, i.e.,

$$
e_{++}(z)=\frac{A}{L} \sin z
$$

This conformal profile corresponds to $e_{12}(z)$ in the HvL model. The two systems differ only by the values of the surface scaling dimensions for the energy: to $\left(x_{\mathrm{e}}^{\mathrm{s}}\right)_{++}=2$ in the model with fixed boundary conditions corresponds $x_{\mathrm{e}}^{\mathrm{s}}=2+2 \alpha$ in the $\mathrm{HvL}$ model. The difference comes from the shift between the two excitation spectra, $\Lambda_{k}=\left(\Lambda_{k}\right)_{++}+\alpha$. Since in both cases the surface perturbation does not change the bulk critical behaviour, the bulk exponents appearing in the profiles are those of the homogeneous Ising quantum chain.

The surface magnetic exponent, $x_{\mathrm{m}}^{\mathrm{s}}$, vanishes for the two problems due to surface order. Thus, if conformal invariance holds as indicated by the numerical data, 
one expects the two magnetization profiles to differ only through the value of their amplitude, which is $\alpha$-dependent in the HvL model and, asymptotically, we have

$$
m(l, \alpha) \sim m_{++}(l)=\left|\begin{array}{ccccc}
1 & 0 & 0 & \ldots & 0 \\
0 & \left(G_{21}\right)_{++} & \left(G_{22}\right)_{++} & \ldots & \left(G_{2 l-1}\right)_{++} \\
\vdots & \vdots & \vdots & & \vdots \\
0 & \left(G_{l 1}\right)_{++} & \left(G_{l 2}\right)_{++} & \ldots & \left(G_{l l-1}\right)_{++}
\end{array}\right|
$$

where $\left(G_{i, j}\right)_{++}$is defined as in (4.9) and the index ++ refers to the homogeneous chain with fixed boundary conditions. This expression has to be compared to (4.8) where $\phi_{0}(1)=\mathrm{O}(1)$ and $\phi_{0}(n)=\mathrm{O}\left(L^{-\alpha}\right)$ when $z=n \pi / L=\mathrm{O}(1)$. All the information about the inhomogeneity is then contained in the non-universal amplitude $A(\alpha)$ which is increasing with $\alpha$, like the surface critical magnetization.

To summarize, we have studied the critical energy and magnetization profiles of the HvL model, using the methods of conformal invariance and through direct analytical and numerical calculations. We have shown that the scaling form of the profiles, resulting from conformal invariance, remains valid for the marginally perturbed system. In the regimes of second- and first-order surface transitions, the profiles keep the same form as for the unperturbed system with free and fixed boundary conditions, respectively, with the HvL values for the surface exponents.

\section{Acknowledgments}

The Laboratoire de Physique des Matériaux is Unité Mixte de Recherche CNRS No 7556. The work of FI has been supported by the Hungarian National Research Fund under grant Nos OTKA TO23642, TO25139, MO28418 and by the Ministery of Education under grant No FKFP 0596/1999.

\section{References}

1] Belavin A A, Polyakov A M and Zamolodchikov A B 1984 Nucl. Phys. B 241333

[2] Cardy J L 1984 Nucl. Phys. B 240514

[3] Turban L 1985 J. Phys. A: Math. Gen. 18 L325

[4] Henkel M and Patkós A 1987 J. Phys. A: Math. Gen. 202199

[5] Burkhardt T W and Iglói F 1990 J. Phys. A: Math. Gen. 23 L633

[6] Iglói F, Berche B and Turban L 1990 Phys. Rev. Lett. 651773

[7] Bariev R Z and Peschel I 1991 J. Phys. A: Math. Gen. 24 L87

[8] Turban L 1991 Phys. Rev. B 447051

[9] Bariev R Z and Peschel I 1991 Phys. Lett. A 153166

[10] Turban L and Berche B 1993 J. Phys. A: Math. Gen. 263131

[11] Hilhorst H J and van Leeuwen J M J 1981 Phys. Rev. Lett. 471188

[12] Blöte H W J and Hilhorst H J 1983 Phys. Rev. Lett. 512015

[13] Burkhardt T W and Guim I 1984 Phys. Rev. B 29508

[14] Burkhardt T W, Guim I, Hilhorst H J and van Leeuwen J M J 1984 Phys. Rev. B 301486

[15] Peschel I 1984 Phys. Rev. B 306783

[16] Blöte H W J and Hilhorst H J 1985 J. Phys. A: Math. Gen. 183039

[17] Bariev R Z 1988 Sov. Phys.-JETP 672170

[18] Iglói F 1990 Phys. Rev. Lett. 643035

[19] Berche B and Turban L 1990 J. Phys. A: Math. Gen. 233029

[20] Peschel I and Wunderling R 1992 Ann. Phys., Lpz. 1125 
[21] Iglói F, Peschel I and Turban L 1993 Adv. Phys. 42683

[22] Burkhardt T W 1982 Phys. Rev. Lett. 48216

[23] Cordery R 1982 Phys. Rev. Lett. 48215

[24] Burkhardt T W 1982 Phys. Rev. B 257048

[25] Turban L and Iglói F 1997 J. Phys. A: Math. Gen. 30 L105

[26] Kogut J B 1979 Rev. Mod. Phys. 51659

[27] Cardy J L 1983 J. Phys. A: Math. Gen. 17 L385

[28] Burkhardt T W and Eisenriegler E 1985 J. Phys. A: Math. Gen. 18 L83

[29] Barber M N, Peschel I and Pearce PA 1984 J. Stat. Phys. 37497

[30] Jordan P and Wigner E 1928 Z. Phys. 47631

[31] Lieb E H, Schultz T D and Mattis D C 1961 Ann. Phys., NY 16406

[32] Pfeuty P 1970 Ann. Phys. Paris 5779

[33] Abramowitz M and Stegun I A 1970 Handbook of Mathematical Functions 9th edn (New York: Dover)

[34] Berche P E, Berche B and Turban L 1996 J. Phys. I 6621 